\title{
Four Gyrophaena Species New to Korea (Coleoptera: Staphylinidae: Aleocharinae)
}

\author{
Yoon-Ho Kim and Kee-Jeong Ahn* \\ Department of Biology, Chungnam National University, Daejeon 305-764, Korea
}

\begin{abstract}
Four Gyrophaena Mannerheim species-Gyrophaena harmandi Fauvel, G. lewisiana Cameron, G. puncticeps Bernhauer and G. rousi Dvoŕák-are reported for the first time in Korea. Redescriptions, habitus illustrations, and line drawings of diagnostic characters are provided.
\end{abstract}

Key words: Coleoptera, Staphylinidae, Aleocharinae, Gyrophaena, Korea

\section{INTRODUCTION}

The genus Gyrophaena Mannerheim contains more than 210 species from the Palaearctic region (Smetana, 2004). Six species have been recorded to date in Korea (Bernhauer, 1936; Yuh et al., 1985; Paśnik, 2001). Biology and diagnostic characters of Gyrophaena were presented by Ashe (1984).

While working on the taxonomy of the Korean Gyrophaena, we found four Gyrophaena species-Gyrophaena harmandi Fauvel, G. lewisiana Cameron, G. puncticeps Bernhauer and $G$. rousi Dvořák-new to the Korean fauna. In this paper, we provide redescriptions, habitus photos, and line drawings of the diagnostic characters. The specimens used in this study are deposited in the Chungnam National University Insect Collection (CNUIC, Daejeon), Korea.

\section{RESULTS}

${ }^{1 * G y r o p h a e n a ~ h a r m a n d i ~ F a u v e l, ~} 1901$ (Figs. 1A, 2) Gyrophaena harmandi Fauvel, 1901: 4; Smetana, 2004: 444.

Material examined. KOREA: Gangwon Prov., Pyeongchanggun, Jinbu-myeon, Odaesan Mt., Sangwonsa, 21 IX 2006, SJ Park, SI Lee, YH Kim, ex mushroom (42주우, CNUIC). Redescription. Length about 2.0-2.2 mm. Body (Fig. 1A) broad and elongate in dorsal aspect. Surface subglossy and slightly pubescent. Body light brown to brown. Head and abdominal tergites VI-VIII dark brown, leg yellow. Body widest at posterior margin of elytra. Head 1.2 times wider

*To whom correspondence should be addressed

Tel: 82-42-821-5492, Fax: 82-42-822-9690

E-mail: kjahn@cnu.ac.kr than long, pronotum 1.4 times wider than long, elytra 1.5 times longer than wide. Head widest across eye, several punctures present. Antennomere 4 about 1.3 times wider than long, 5-6 about as long as wide, 7-10 about 1.1 times wider than long, 11 about 1.6 times longer than wide (Fig. 2A). Pronotum widest at middle, anterior margin straight, posterior margin broadly round (Fig. 2B). Postero-lateral angle of elytra slightly sinuate. Tergites III-VI transversely impressed. Male tergite VII with one distinct tubercle at postero-medial area (Fig. 2C). Male tergite VIII with two lateral pointed processes and posterior margin slightly pointed medially (Fig. 2D). Posterior margin of male sternite VIII truncate (Fig. 2E). Posterior margin of female sternite VIII slightly round (Fig. 2F). Median lobe cylindrical and asymmetrical, apical process of median lobe slightly curved (Fig. $2 \mathrm{H})$. Flagellum long and coiled. Apical lobe of paramere as in Fig. 2G. Spermatheca as in Fig. 2I.

Remarks. Gyrophaena harmandi can be easily distinguished from other Korean Gyrophaena species by the following features: male tergite VII with one tubercle as in Fig. 2C, male tergite VIII with two processes as in Fig. 2D, and the structure of median lobe as in Fig. $2 \mathrm{H}$.

Distribution. Korea and Japan.

\footnotetext{
2*Gyrophaena lewisiana Cameron, 1933 (Figs. 1B, 3) Gyrophaena lewisiana Cameron, 1933: 175; Smetana, 2004:
} 444.

Material examined. Syntype, 1, labeled as follows: Japan, Settsu, Katsuoji, J.E.A. Lewis, M. Cameron. Bequest. B. M. 1955-147, G. lewisiana Cam., Gyrophaena lewisiana Cam., P.M. Hammond det. 1973 syntype; KOREA: Chungnam Prov., Gongju-city, Banpo-myeon, Sangsin-ri, Mt. Gyeryongsan, 26 VIII 2001, MH Kim, ex mushroom (72 우우, CNUIC);

$1 *$ 어리납작버섯반날개 (신칭), ${ }^{2}$ 가는납작버섯반날개 (신칭) 

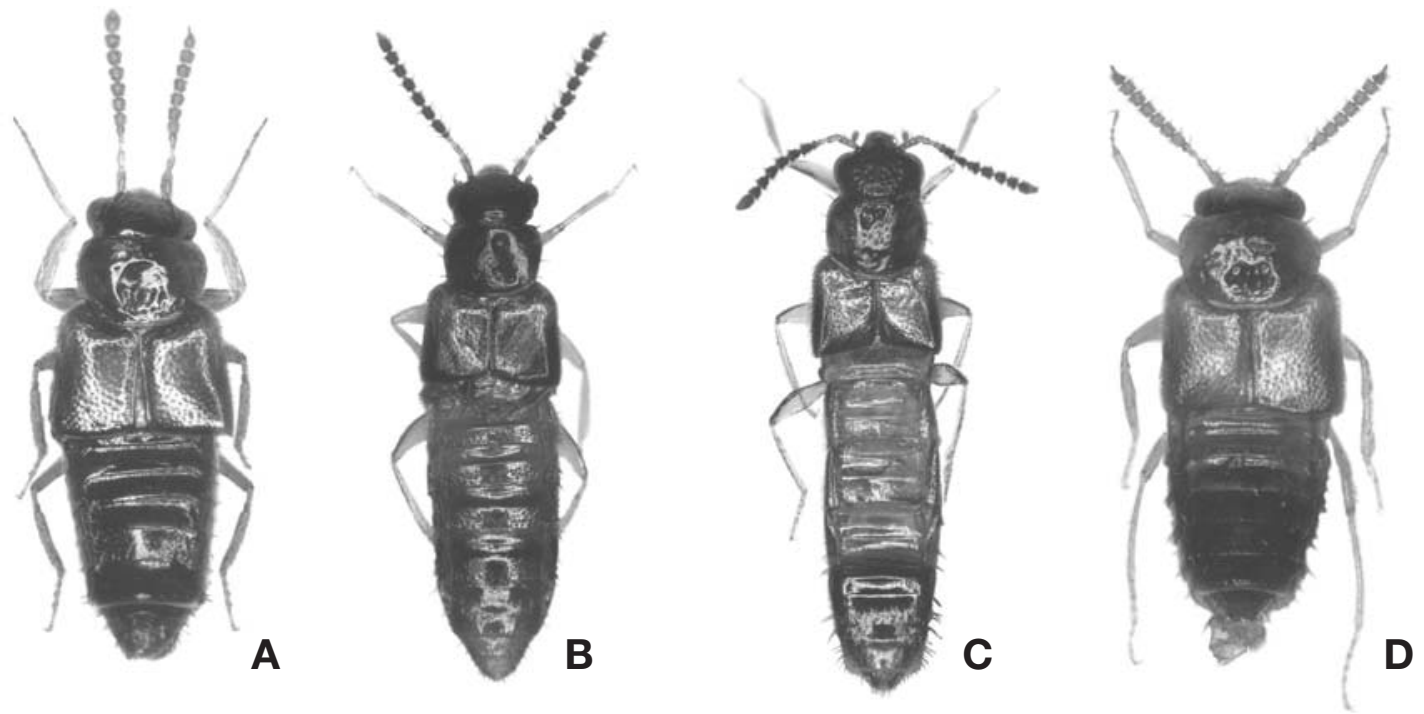

Fig. 1. A, Gyrophaena harmandi, $2.2 \mathrm{~mm}$; B, Gyrophaena lewisiana, $2.3 \mathrm{~mm}$; C, Gyrophaena puncticeps, 2.6 mm; D, Gyrophaena rousi, $1.7 \mathrm{~mm}$.

Chungnam Prov., Daejeon-city, Mt. Sikjangsan, Seocheon park, 30 VII 2000, MH Kim, ex mushroom (24 이 우, CNUIC); Chungbuk Prov., Boeun-gun, Mt Sokrisan, 25 VII 1999, MH Kim ex mushroom (94주우, CNUIC).

Redescription. Length about 2.2-2.5 mm. Body (Fig. 1B) broad and elongate in dorsal aspect. Surface subglossy and slightly pubescent. Body light brown to brown. Head, abdominal tergites VI-VIII and antennomeres 4-11 dark brown, antennomeres 1-3 and leg yellow. Body widest at posterior margin of elytra. Head 1.2 times wider than long, pronotum 1.3 times wider than long, elytra 1.4 times longer than wide. Head widest across eye, several punctures present. Antennomere 4 about 1.4 times wider than long, 5 about 1.4 times longer than wide, 6-7 about 1.3 times longer than wide, 8 about 1.2 times longer than wide, 9 about 1.1 times longer than wide, 10 about as long as wide, 11 about 1.9 times longer than wide (Fig. 3A). Pronotum widest at middle, anterior margin and posterior margin broadly round (Fig. 3B). Postero-lateral angle of elytra not sinuate. Tergites III-VI transversely impressed. Male tergite VIII with two lateral pointed processes and posterior margin truncate (Fig. 3C). Posterior margin of male sternite VIII round (Fig. 3D). Posterior margin of female sternite VIII convex, more or less round (Fig. $3 \mathrm{E})$. Median lobe cylindrical and asymmetrical, apical process of median lobe slightly curved (Fig. 3G). Flagellum long and coiled. Apical lobe of paramere as in Fig. 3F. Spermatheca as in Fig. $3 \mathrm{H}$.

Remarks. Gyrophaena lewisiana is similar to G. puncticeps, but can be distinguished by the following features: posterior margin of pronotum broadly round as in Fig. 3B and male tergite VIII with two processes as in Fig. 3C.

Distribution. Korea and Japan.

1*Gyrophaena puncticeps Bernhauer, 1907 (Figs. 1C, 4) Gyrophaena puncticeps Bernhauer, 1907: 389; Smetana, 2004: 445.

Material examined. KOREA: Chungnam Prov., Gongju-city, Gyeryong-myeon, Yanghwa-ri, Shinwonsa, 6 VIII 2001, MH Kim, ex mushroom (68 이 우, CNUIC); Chungnam Prov., Gongju-city, Gyeryong-myeon, Jungjang-ri, Mt. Gyeryongsan, Gapsa, 12 VIII 2000, MH Kim, ex mushroom (18 우우, CNUIC); Chungbuk Prov., Boeun-gun, Mt. Sokrisan, 15 VIII 1999, MH Kim, ex mushroom (21 주우, CNUIC); Gyeongbuk Prov., Sangju-city, Hwanam-myeon, Imgok-ri, Mt. Cheontaeksan, 27 VII 2001, MH Kim, ex mushroom (28 ð 우, CNUIC).

Redescription. Length about 2.6-2.9 mm. Body (Fig. 1C) moderate and elongate in dorsal aspect. Surface subglossy and slightly pubescent. Body light brown to brown. Head, abdominal tergites VI-VIII and antennomeres 4-11 dark brown, antennomeres 1-3 and leg yellow. Body widest at posterior margin of elytra. Head 1.1 times wider than long, pronotum 1.2 times wider than long, elytra 1.6 times longer than wide. Head widest across eye, many distinct punctures present. Antennomere 4 about 1.2 times wider than long, 5 about 1.2 times longer than wide, 6 about 1.3 times longer than wide, 7-9 about 1.1 times longer than wide, 10 as long

\footnotetext{
1 *긴납작버섯반날개 (신칭)
} 

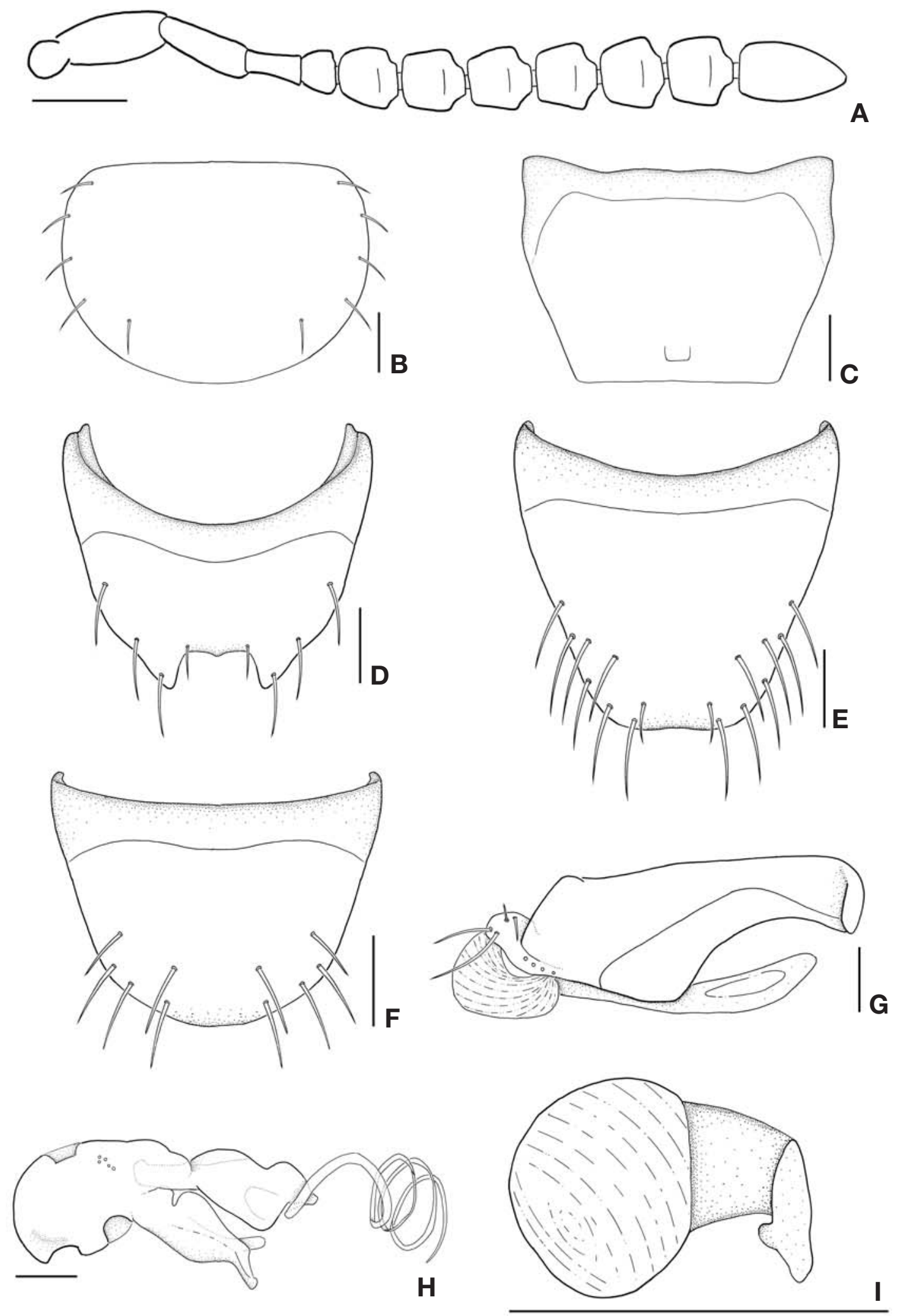

Fig. 2. Gyrophaena harmandi. A, antenna, dorsal aspect; $B$, pronotum, dorsal aspect; $C$, male tergite VII, dorsal aspect; $D$, male tergite VIII, dorsal aspect; E, male sternite VIII, ventral aspect; F, female sternite VIII, ventral aspect; G, paramere, lateral aspect; $\mathrm{H}$, median lobe, lateral aspect; I, spermatheca, lateral aspect. Scale bars $=0.1 \mathrm{~mm}$. 

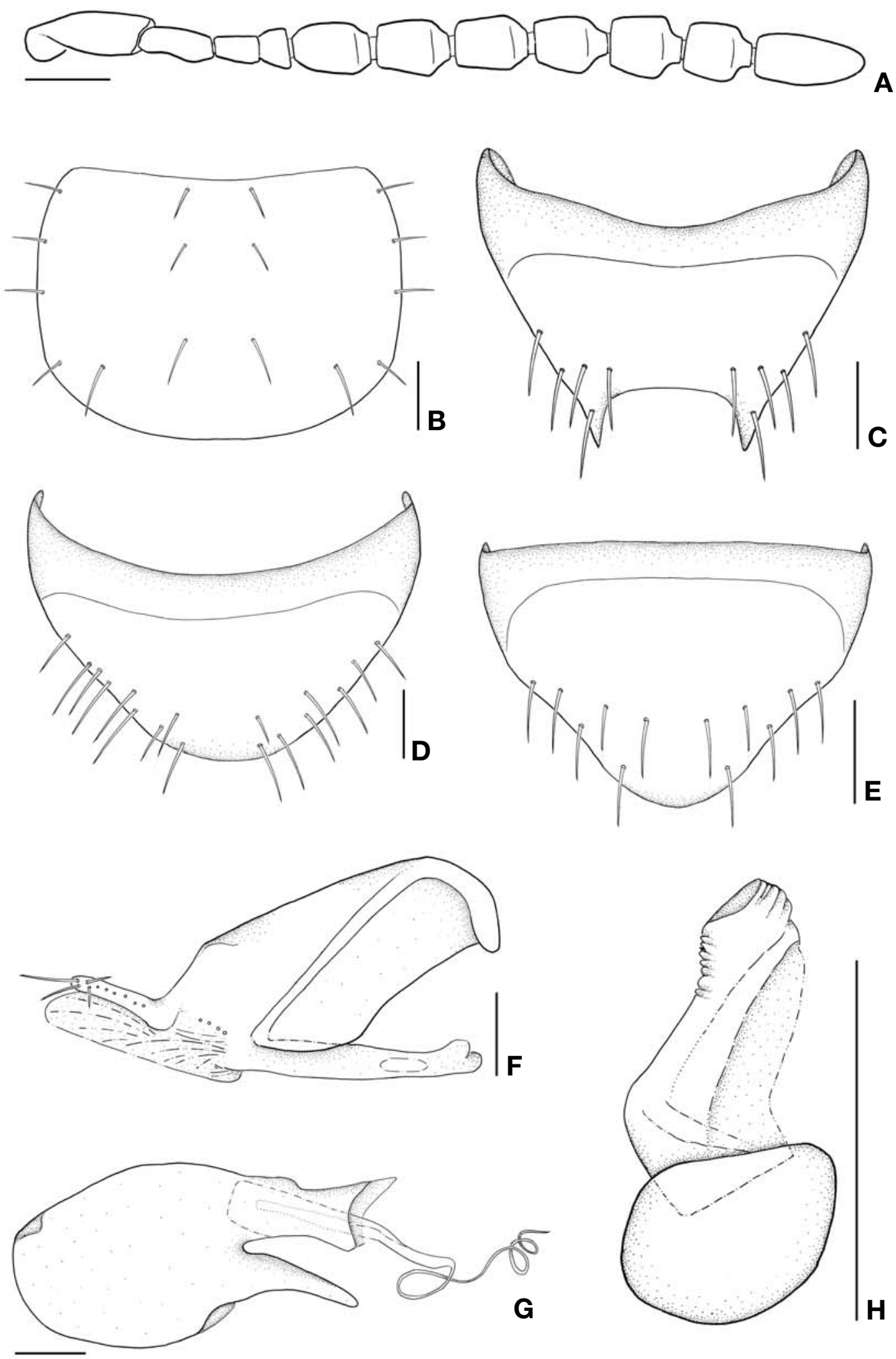

Fig. 3. Gyrophaena lewisiana. A, antenna, dorsal aspect; $B$, pronotum, dorsal aspect; $C$, male tergite VIII, dorsal aspect; D, male sternite VIII, ventral aspect; E, female sternite VIII, ventral aspect; F, paramere, lateral aspect; $G$, median lobe, lateral aspect; $H$, spermatheca, lateral aspect. Scale bars $=0.1 \mathrm{~mm}$. 

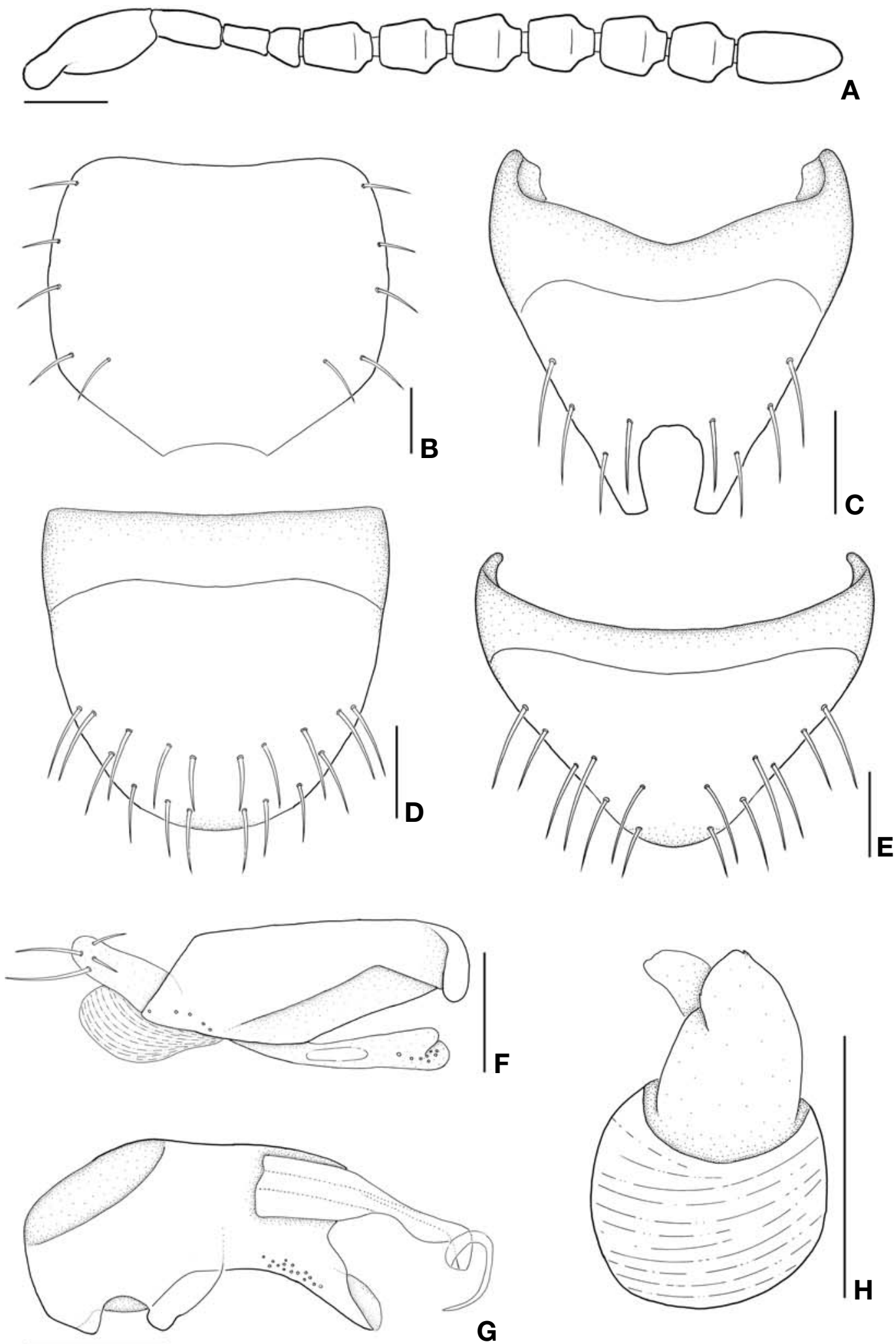

Fig. 4. Gyrophaena puncticeps. A, antenna, dorsal aspect; B, pronotum, dorsal aspect; C, male tergite VIII, dorsal aspect; D, male sternite VIII, ventral aspect; E, female sternite VIII, ventral aspect; F, paramere, lateral aspect; $G$, median lobe, lateral aspect; $H$, spermatheca, lateral aspect. Scale bars $=0.1 \mathrm{~mm}$. 

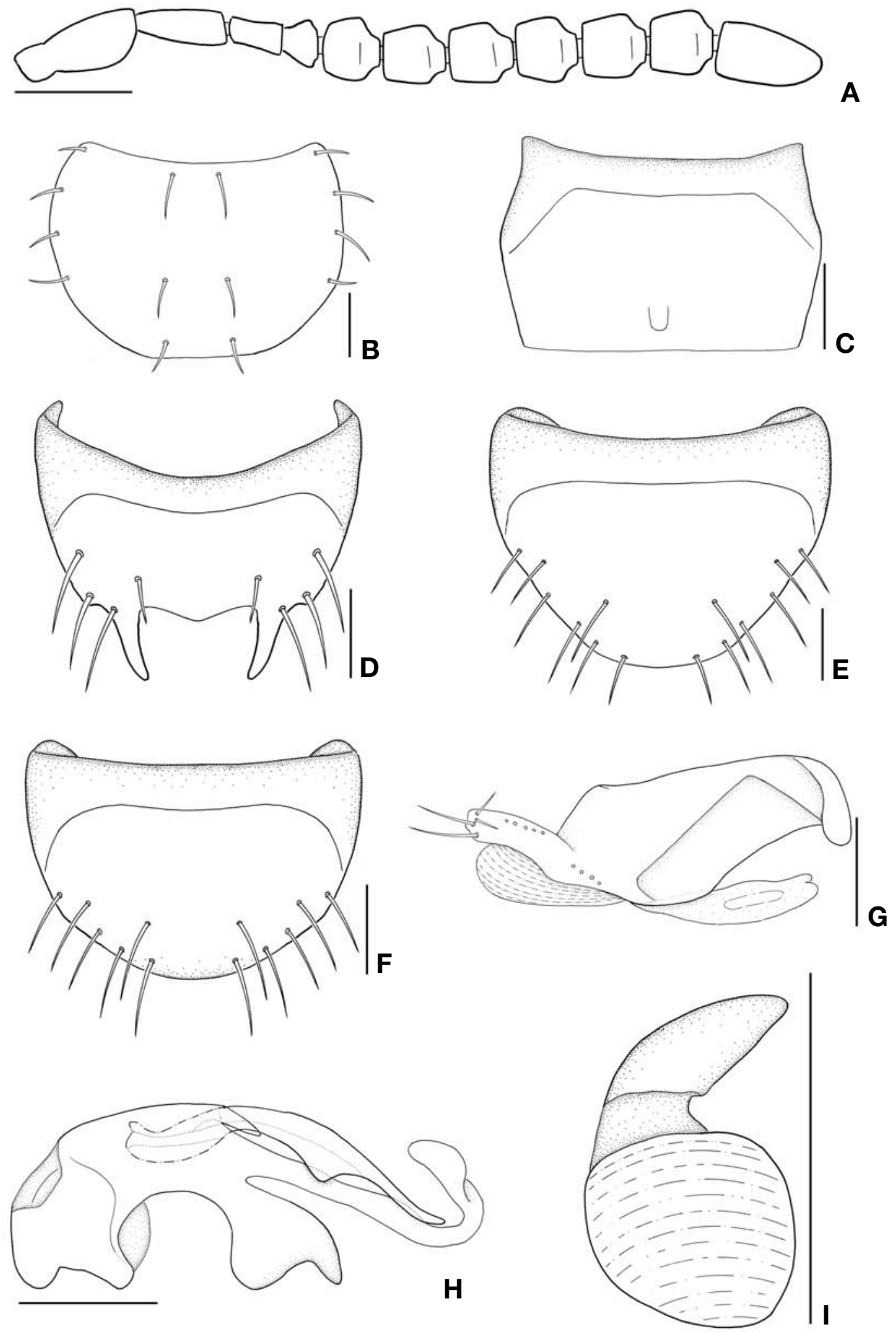

Fig. 5. Gyrophaena rousi. A, antenna, dorsal aspect; B, pronotum, dorsal aspect; C, male tergite VII, dorsal aspect; D, male tergite VIII, dorsal aspect; E, male sternite VIII, ventral aspect; F, female sternite VIII, ventral aspect; G, paramere, lateral aspect; $H$, median lobe, lateral aspect; I, spermatheca, lateral aspect. Scale bars $=0.1 \mathrm{~mm}$. 
as wide, 11 about 1.7 times longer than wide (Fig. 4A). Pronotum widest at middle, anterior margin broadly round, posterior margin as in Fig. 4B. Postero-lateral angle of elytra not sinuate. Tergites III-VI transversely impressed. Male tergite VIII with two lateral truncated processes (Fig. 4C). Posterior margin of male sternite VIII more or less round (Fig. 4D). Posterior margin of female sternite VIII convex, more or less round (Fig. 4E). Median lobe cylindrical and asymmetrical, apical process of median lobe slightly curved (Fig. 4G). Flagellum short and not coiled. Apical lobe of paramere as in Fig. 4F. Spermatheca as in Fig. 4, H.

Remarks. Gyrophaena puncticeps is similar to G. lewisiana, but can be distinguished by the following features: distinct structure of pronotum as in Fig. 4B and male tergite VIII with two broad processes as in Fig. 4C.

Distribution. Korea and Japan.

1*Gyrophaena rousi Dvořák, 1966 (Figs. 1D, 5)

Gyrophaena rousi Dvořák, 1966: 1; Lohse, 1974: 29; Smetana, 2004: 445.

Material examined. KOREA: Chungnam Prov., Gongju-city, Mt. Gyeryongsan, Gapsa, 2 VII 2000, MH Kim, ex mushroom (10주우, CNUIC); Gangwon Prov., Pyeongchang-gun, Jinbumyeon, Mt. Odaesan, Chongmyeolbogung, 8 VII 1998, MH Kim ex mushroom (3주우, CNUIC).

Redescription. Length about 1.6-1.9 mm. Body (Fig. 1D) broad and elongate in dorsal aspect. Surface subglossy and slightly pubescent. Body light brown to brown. Head and abdominal tergites V-VIII dark brown, leg yellow. Body widest at posterior margin of elytra. Head 1.1 times wider than long, pronotum 1.4 times wider than long, elytra 1.5 times longer than wide. Head widest across eye, several punctures present. Antennomere 4 about 1.1 times wider than long, 5-8 about as long as wide, 9-10 about 1.1 times wider than long, 11 about 1.5 times longer than wide (Fig. 5A). Pronotum widest at middle, anterior margin straight, posterior margin broadly round (Fig. 5B). Postero-lateral angle of elytra slightly sinuate. Tergites III-VI transversely impressed. Male tergite VII with one distinct tubercle at postero-medial area (Fig. 5C). Male tergite VIII with two lateral processes and posterior margin slightly protruded medially (Fig. 5D). Posterior margin of male sternite VIII slightly round (Fig. 5E). Posterior margin of female sternite VIII slightly round (Fig. 5F). Median lobe cylindrical and asymmetrical, apical process of median lobe bifid and curved (Fig. 5H). Flagellum short and not coiled. Apical lobe of paramere as in Fig. 5G. Spermatheca as in Fig. 5I.

Remarks. Gyrophaena rousi is similar to G. triquetra, but can be distinguished by the following features: antennomeres 8-10 transverse as in Fig. 5A, male tergite VII with one tubercle as in Fig. 5C, and male tergite VIII with two processes as in Fig. 5D.

Distribution. Korea, Central Europe, England, Germany, Slovakia and Sweden.

\section{ACKNOWLEDGEMENTS}

We thank Dr. R. Booth (The Natural History Museum, London), Dr. J. H. Boone (Field Museum of Natural History, Chicago) and Dr. Y. Gèrard (Institut Royal des Sciences Naturelles de Belgique, IRSNB, Bruxelle) for the loan of type specimens. This research was supported by a grant (NIBR 0741800-1844-326-260-00) from the Ministry of Environment of the Korean Government.

\section{REFERENCES}

Ashe, J.S., 1984. Generic revision of the subtribe Gyrophaenina (Coleoptera: Staphylinidae: Aleocharinae) with a review of the described subgenera and major features of evolution. Quaest. Entomol., 20: 129-349.

Bernhauer, M., 1907. Zur Staphylinidenfauna von Japan. Verh. K.-K. Zool.-Bot. Ges. Wien., 57: 371-414.

Bernhauer, M., 1936. Neuheiten der palaearktischen Staphylinidenfauna. Publ. Mus. Entomol. "Pietro Rossi" Duino, 1: 237-254, 303-325.

Cameron, M., 1933. New species of Staphylinidae (Col.) from Japan. The Entomol. Mon. Mag., 69: 168-175, 208-219.

Dvořák, M., 1966. Gyrophaena rousi sp. n., eine neue Staphyliniden-Art aus der Slowakei (Col., Staphylinidae). Annot. Zool. Bot., 30: 1-4.

Fauvel, A., 1901. Staphylinides nouveaux du Japon. Rev. Entomol., 20: 2-5.

Lohse, G.A., 1974. Die Käfer Mitteleuropas. Bd. 5. Staphylinidae II (Hypocyphtinae und Aleocharinae), Pselaphidae. Goeke \& Evers, Krefeld, pp. 1-381.

Paśnik, G., 2001. The North Korean Aleocharinae (Coleoptera, Staphylinidae) diversity and biogeography. Acta Zool. Cracov., 44(3): 185-234.

Smetana, A., 2004. Family Staphylinidae: Aleocharinae. In Löbl, I. and A. Smetana, eds., Catalogue of Palaearctic Coleoptera, Volume 2, Hydrophiloidea, Histeroidea, Staphylinoidea. Apollo Books. Stenstrup, Denmark, pp. 353-494.

Yuh, J.H., W.H. Paik, Y.J. Kwon and S.M. Lee, 1985. Check list of rove beetles from Korea (Coleoptera: Staphylinidae). Ins. Koreana, 5: 223-255.

Received April 22, 2009 Accepted May 10, 2009

\footnotetext{
$1 *$ 둥글납작버섯반날개
} 